\title{
Correction to: Effectiveness of Exercise Interventions to Improve Postural Control in Older Adults: A Systematic Review and Meta- Analyses of Centre of Pressure Measurements
}

\author{
Daniel C. Low ${ }^{1}$ Gregory S. Walsh ${ }^{1} \cdot$ Marco Arkesteijn ${ }^{1}$
}

Published online: 20 October 2017

(c) Springer International Publishing AG 2017

Correction to: Sports Med (2017) 47:101-112

DOI 10.1007/s40279-016-0559-0

Page 103, Table 1: The cell entry in the 'Terms used' column which previously read:

"Centre of pressure OR center of pressure OR COP OR postural sway OR postural stability OR postural control OR balance OR force plat*”

should read:

"Randomised control* OR randomized control* OR RCT AND falls OR falling OR faller*”
Page 103, Table 1: The cell entry in the 'Terms used' column which previously read:

"Randomised control* OR randomized control* OR RCT AND falls OR falling OR faller*”

should read:

"Centre of pressure OR center of pressure OR COP OR postural sway OR postural stability OR postural control OR balance OR force plat*"

The online version of the original article can be found under doi:10.1007/s40279-016-0559-0.

Daniel C. Low

dal38@aber.ac.uk

Institute of Biological, Environmental and Rural Sciences, (IBERS), Aberystwyth University, Carwyn James Building, Penglais Campus, Aberystwyth SY23 3FD, UK 